\title{
Radio Evidence for Non-isotropic Outflows from Hot Stars
}

\author{
Stephen White \\ Dept. of Astronomy, University of Maryland, \\ College Park, MD 20742
}

\begin{abstract}
Radio diagnostics for outflows from hot stars are reviewed and three well-studied examples (WR 140, $\eta$ Car and MWC 349) are discussed in some detail. The three systems are very different and their radio properties are diverse, but in each case, the radio data provide strong evidence for the presence of equatoriallyenhanced outflows.
\end{abstract}

\section{Introduction}

Radio observations provide a powerful tool for the study of hot stars. Radio emission can penetrate the clouds of dust which tend to obscure the locations of massive stars, and are capable of high spatial resolution. On the other hand, there are some notable limitations to what radio observations can do. We first discuss the general properties of radio emission from hot stars and then discuss three examples in more detail.

Two very different emission mechanisms are responsible for radio emission from hot stars, and both arise in their outflows. The most common is thermal free-free or bremsstrahlung, which is responsible for the classic stellar-wind radio emission. The opacity for this mechanism varies as $n_{e}^{2} T^{-1.5} \nu^{-2}$, where $n_{e}$ is the electron density, $T$ the electron temperature and $\nu$ the radio frequency. The fact that the opacity decreases as frequency increases, while density in an outflow decreases with radius, leads to a fundamental property of free-free radio emission from stellar winds: higher frequencies probe deeper into the stellar wind. For a constant-velocity wind $\left(n_{e} \propto r^{-2}\right)$ the radius of the optically thick surface, which limits how deeply we can see, scales with frequency as $\nu^{-0.7}$. The combination of this scaling of optically-thick source dimension with frequency and the $\nu^{+2}$-dependence of the black-body emission law produces the classic $\nu^{0.6}$ spectrum of a constant-velocity freefree emitting stellar wind (Olnon 1975, Panagia \& Felli 1975, Wright \& Barlow 1975). This law does not require a spherically-homogeneous outflow: the outflow can be asymmetric and maintain the $\nu^{0.6}$ spectrum as long as wind motion is radial and the temperature and ionization state remain unchanged with radius (Schmid-Burgk 1982).

The second mechanism is nonthermal synchrotron emission, more familiar from supernova remnants and quasars. It is important in hot stars because of 
the facility with which shocks can form in the powerful winds: the characteristic speed of a hot star wind is generally in excess of the ambient sound speed in the wind, so that any significant velocity fluctuation, such as a fast knot overtaking a slower one or two winds colliding, has the potential to result in a shock. Once a shock forms, electron acceleration apparently takes place (by an as yet not completely understood mechanism). A high Mach-number shock produces a characteristic power-law energy distribution of spectral index -2 , which results in a $\nu^{-0.5}$ radio flux spectrum (in the optically-thin limit believed appropriate to these sources). A detailed model for nonthermal emission from a single-star wind carrying random shocks was worked out by Rick White (1985). Note that the acceleration must take place at some distance from the star: the powerful radiation field of a hot star can quench shock acceleration close to the star where the inverse-Compton mechanism depletes energy from a high-energy electron faster than a shock can supply it (Chen \& White 1994). This is a mild problem for models in which the magnetic field in the synchrotron source is a stellar field carried out by the wind, since it will diminish with distance from the star. An alternative is for the magnetic field also to be generated in the shocks as a byproduct of the plasma turbulence there.

\section{$2 \quad$ MWC 349}

MWC 349 is one of the most extreme galactic members of the class of luminous B stars showing forbidden emission lines, known as B[e] stars. These stars are characterized by very strong Balmer lines, forbidden FeII lines and very strong emission from hot dust at infrared wavelengths. These features are assumed to derive from the combination of a very dense equatorial disk, which provides the Balmer emission and the dust, together with a powerful ionized polar outflow which is responsible for the forbidden lines (e.g., see the review by Conti 1997).

MWC 349's radio spectral index of +0.6 was the prime evidence for the correctness of the stellar-wind outflow interpretation of the radio emission. However, the original model of a spherically-symmetric outflow had to be abandoned when VLA observations showed remarkable structure in the radio emission (Cohen et al. 1985, White \& Becker 1985). A high-spatial-resolution image of the $8 \mathrm{GHz}$ radio continuum is shown in Fig. 1. It is seen to break up into two sets of curved "horns" on either side of the star with much weaker emission between. The peak brightness temperature in the horns is $10^{4} \mathrm{~K}$, while it drops to $4400 \mathrm{~K}$ at the inferred position of the star. The interpretation of this image is that the outflow from MWC 349 has low velocity but high density in the equatorial plane (the low-brightness region between the horns), and higher velocity but lower density in the polar directions (the directions of the horns). The density is so high in the equatorial plane that the gas there has completely recombined and is neutral, while in the polar directions 


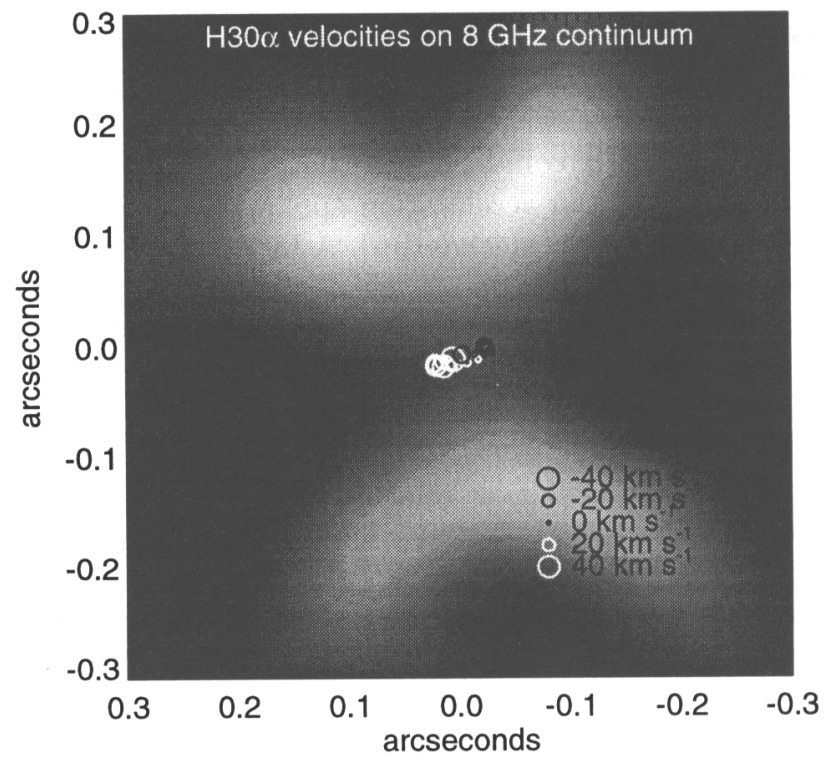

Fig. 1. The positions of different velocities in the masing $232 \mathrm{GHz} H 30 \alpha$ recombination line (White et al. 1998) plotted over a greyscale display of the $8 \mathrm{GHz}$ VLA continuum image of MWC 349 (data from Rodríguez \& Bastian 1994), showing the relationship of the line emission in the disk to the continuum emission from the polar outflow. Note that the bulk of the material in the equatorial plane is believed to be neutral and hence produces little microwave emission, whereas the polar outflow is believed to be ionized.

the gas is still ionized and thus can be optically thick at the temperature characteristic of a photo-ionized wind.

MWC 349 is also famous among radio astronomers as the first source to show masing (and recently, lasing) recombination lines (Martín-Pintado et al. 1989, Strelnitski et al. 1996). It has long been recognized that population inversion occurs in Hydrogen level populations in photoionized sources, due to the fact that a recombining electron generally re-enters the energy-level ladder at the top, and the rate of transitions increases as it drops down the ladder to lower energy levels. However, population inversion sufficient to produce a significant amplification was first seen in MWC 349, and seems to occur there for all $\Delta n=1$ transitions with $n<36(f>135 \mathrm{GHz})$. At 232 $\mathrm{GHz}$ the $\mathrm{H} 30 \alpha$ line shows two very strong features at $+32 \mathrm{~km} \mathrm{~s}^{-1}$ and -16 $\mathrm{km} \mathrm{s}^{-1}$, and Fig. 1 also shows the positions of different velocities within this recombination line, from a recent observation with the BIMA array (White et al. 1998). These data confirm that the masing recom line arises in the equatorial plane, not in the bipolar horns where the continuum emission is strongest. The position-velocity diagram across the disk is consistent with 
the masing emission arising at a constant-radius surface, presumably where the continuum becomes optically thick. Such masing lines promise to be a valuable diagnostic for hot star winds in the future: they are strongest at millimeter and far infra-red wavelengths where dust obscuration is low and high spatial resolution can be achieved, and the line data can be used to derive kinematic information for stellar outflows.

\section{$3 \quad$ WR $140=$ HD 193793}

This remarkable system demonstrates that a wealth of information on the outflows from hot stars can be gained even from radio data in from a system which is spatially unresolved. WR 140 consists of a (secondary) WC7 WolfRayet (WR) star and an O4-5 star (primary) in a highly elliptical orbit with a period of 7.9 years. Early radio surveys of WR stars revealed that WR 140 was a strong and interesting source with a nonthermal spectrum, and subsequent observations have only reinforced this view (e.g., Becker \& White 1985, Williams et al. 1990, White \& Becker 1995). However, at a distance of $1.3 \mathrm{kpc}$ the major axis of the orbit, $29 \mathrm{AU}$, subtends just 2 millarcseconds, which is barely feasible with VLBI techniques, and to date only radio light curves are available.

White \& Becker (1995) monitored the radio emission from WR 140 at three frequencies $(1.4,5$ and $15 \mathrm{GHz})$ with the VLA every month for 8 years. The light curves show a wealth of structure. The basic model is that the nonthermal emission arises due to synchrotron emission from energetic electrons accelerated in the shock where the powerful winds from the two stars collide. There is also some free-free emission from the winds, and the nonthermal radio emission is modulated by free-free absorption as the location of the shock changes with respect to the winds. The shock strengthens near periastron and weakens near apastron. The orientation of the orbit is such that the $\mathrm{O}$ star is closest to us during most of the orbit; the WC7 star passes between us and the $\mathrm{O}$ star just after periastron, at phase 0.01 . The $\mathrm{O}$ star wind has a terminal velocity of $3100 \mathrm{~km} \mathrm{~s}^{-1}$ with $\dot{M}=1.8 \times 10^{-6} \mathrm{M}_{\odot} \mathrm{yr}^{-1}$, while the WR wind has a velocity of $2600 \mathrm{~km} \mathrm{~s}^{-1}$ with $\dot{M}=6 \times 10^{-5} \mathrm{M}_{\odot} \mathrm{yr}^{-1}$. In principle, to model this system one needs to know all the physical propoerties of the binary orbit and the winds, and understand the shock physics and its variation with stellar separation, electron acceleration at the shock, the source of magnetic fields and absorption in the wind; conversely, all these phenomena affect the radio light curves.

The light curves are plotted in Figure 2. Free-free absorption should be largest at the lowest frequency and the $1.5 \mathrm{GHz}$ light curve is consistent with this expectation: it shows no significant flux except for a narrow peak near phase 0.8 . At $5 \mathrm{GHz}$ the peak is much broader, with only weak emission just after phase zero when the strong wind of the WR star is between us and the wind-interaction region. The $15 \mathrm{GHz}$ emission, which should suffer the least 


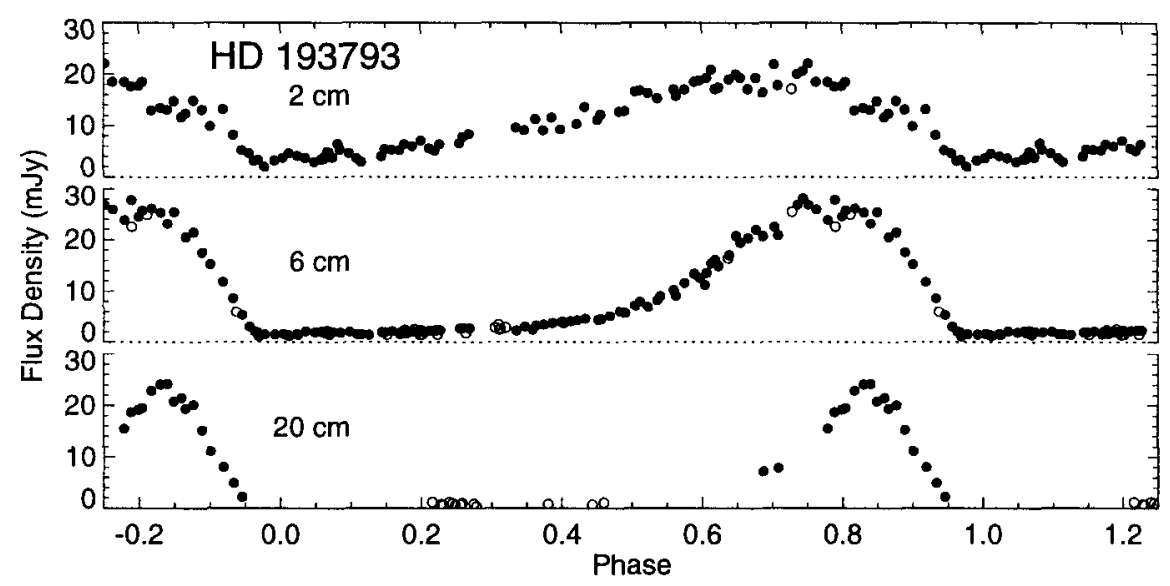

Fig. 2. The radio light curve for WR 140 plotted against orbital phase over the 7.9-year orbit at each of three wavelengths. Filled circles are VLA data taken every month by White \& Becker (1995); open circles represent other data compiled by Williams et al. (1990) covering more than one cycle, indicating that the light curve repeats almost perfectly from one cycle to the next. (Figure provided by Rick White.)

absorption and therefore best represent the intrinsic emission profile, peaks at phase 0.70 , somewhat earlier than the $1.5 \mathrm{GHz}$ peak: between phases 0.70 and 0.85 the $15 \mathrm{GHz}$ flux drops while the $1.5 \mathrm{GHz}$ emission rises. From phase 0 to 0.5 , the spectral index is positive, consistent with thermal free-free emission.

The fact that there is any $1.5 \mathrm{GHz}$ emission detected at all is difficult to explain, since the parameters for the WR wind imply that it should be optically thick at $1.5 \mathrm{GHz}$ well outside the binary orbit. Williams et al. (1990) showed that a model with spherically-symmetric winds could not explain the light curves and argued that the shadow of the $O$ star creates a cavity in the WR wind where the opacity is greatly reduced. White \& Becker (1995) argued that even the $\mathrm{O}$ star wind will be optically thick and absorb any $20 \mathrm{~cm}$ emission behind it; they suggest instead that the WR wind is confined to an equatorial plane inclined with respect to the orbital plane. In this geometry the WR wind sweeps across the position of the $\mathrm{O}$ star twice per orbit; one of these occasions is presumably at phase 0.70 , where the radio flux is maximum. Thus in this system an equatorially enhanced wind for the WR star is not resolved directly but is inferred from the light curves.

\section{$4 \eta$ Carinae}

$\eta$ Carinae as a radio source shows an interesting amalgam of the properties of the two previous examples. Like MWC 349, the radio emission from $\eta$ Car is entirely thermal free-free emission (ignoring some nonthermal emission from 


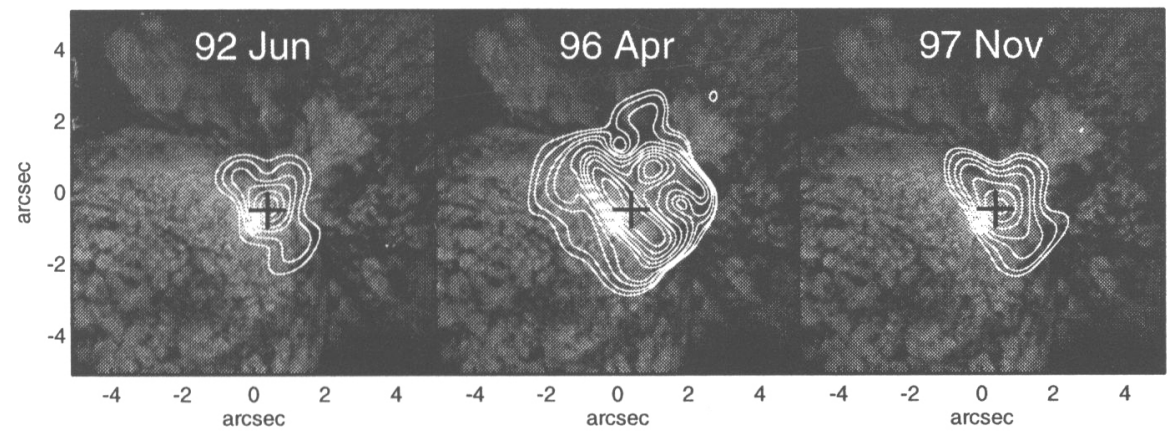

Fig. 3. Contours of $8 \mathrm{GHz}$ radio emission overlaid on the HST image of the core of the $\eta$ Carina nebula, at three different epochs. The resolution of the radio data is of order $0.3^{\prime \prime}$. The bipolar lobes of the Homunculus nebula are seen in the lower-left and upper-right corners; the "equatorial skirt" lies just above the diagonal running from the upper left corner to the bottom right corner.

features in the outer nebula) but like WR 140 the emission is apparently strongly influenced by the binary nature of the system.

Figure 3 shows how the spatial distribution of radio emission from $\eta$ Car evolves over the 5.5 year cycle discovered by Damineli (1996). The period is believed to represent the orbital period of a binary system consisting of an LBV star and an $\mathrm{O}$ star in a highly eccentric orbit, with our line of sight, when projected onto the orbital plane, coinciding almost exactly with the major axis of the orbit (Damineli, Conti \& Lopes 1997, Davidson 1997). The radio light curve shows a large variation over the 5.5-year cycle, from 0.7 $\mathrm{Jy}$ at $8.8 \mathrm{GHz}$ in 1992 June (corresponding to periastron for the binary, i.e., the time of minimum excitation in the optical spectrum noted by Damineli) to $3 \mathrm{Jy}$ in 1995 (near apastron) and back down to $0.9 \mathrm{Jy}$ in January 1998 (periastron again).

The images show that the changes in the radio flux are due to the appearance of extended emission which is not present at periastron (White et al. 1994, Duncan et al. 1995, Duncan, White \& Lim 1997). The radio spectrum of all of this emission is consistent with thermal free free emission: the brightest sources have brightness temperatures of $10^{4} \mathrm{~K}$, and the weaker extended emission has the flat spectrum expected of optically-thin free-free emission. In addition, we observe recombination-line emission from the extended emission, implying photo-ionized gas. The implication is that all of this additional emission comes from gas which is neutral when the binary is at periastron, and ionized when the system is near apastron.

The location and kinematics of the gas indicate its nature: it lies almost entirely to the north-west of the star, and the recombination line data indicate that all the gas is blue-shifted with respect to the systemic velocity. The 
lobe of the Homunculus nebula which is projected to the north-west of the star is known to be receding from us, so the velocity of the radio-emitting gas is inconsistent with a location there: instead, it must be in the equatorial skirt. Material in the skirt which appears projected to the north-west of the star should indeed be moving towards us. We argue that the gas in the outflow from $\eta$ Car (estimated to be $10^{-3} \mathrm{M}_{\odot} \mathrm{yr}^{-1}$ at $500 \mathrm{~km} \mathrm{~s}^{-1}$ ) is so dense that it recombines quite close to the star(s), and hence most of the gas surrounding the system is neutral. The ionizing photons from the LBV star never penetrate very far through its massive outflow. Near periastron the hot companion star is also enveloped in the outflow of the LBV star, but away from periastron the LBV is on the far side of the $O$ star and ionizing photons from the $\mathrm{O}$ star can reach gas in the equatorial skirt in our direction, ionizing it and causing it to produce radio emission. When periastron approaches the LBV wind again cuts off the ionizing flux from the companion and the neutral gas recombines and vanishes from the radio images.

\section{References}

Becker, R. H., \& White, R. L. 1985, ApJ, 297, 649.

Chen, W., \& White, R. L. 1994, Ap\&SS, 221, 259.

Cohen, M., Bieging, J. H., Dreher, J. W., \& Welch, W. J. 1985, ApJ, 292, 249.

Conti, P. S. 1997, in Luminous Blue Variables: Massive Stars in Transition, ed.

A. Nota \& H. J. G. L. M. Lamers (San Francisco: Astron. Soc. Pac.), p. 161.

Damineli, A. 1996, ApJL, 460, L49.

Damineli, A., Conti, P. S., \& Lopes, D. F. 1997, New Astron., 2, 107.

Davidson, K. 1997, New Astron., 2, 387.

Duncan, R. A., White, S. M., \& Lim, J. 1997, MNRAS, 290, 680.

Duncan, R. A., White, S. M., Lim, J., Nelson, G. J., Drake, S. A., \& Kundu, M. R. 1995, ApJL, 441, L73.

Martín-Pintado, J., Bachiller, R., Thum, C., \& Walmsley, C. M. 1989, A\&A, 215, L13.

Olnon, F. M. 1975, A\&A, 39, 217.

Panagia, N., \& Felli, M. 1975, A\&A, 39, 1.

Rodríguez, L. F., \& Bastian, T. S. 1994, ApJ, 428, 324.

Schmid-Burgk, J. 1982, A\&A, 108, 169.

Strelnitski, V. S., Haas, M. R., Smith, H. A., Erickson, E. F., Colgan, S. W. J., \& Hollenbach, D. J. 1996, Science, 272, 1459.

White, R. L. 1985, ApJ, 289, 698.

White, R. L., \& Becker, R. H. 1985, ApJ, 297, 677.

White, R. L., \& Becker, R. H. 1995, ApJ, 451, 352.

White, S. M., Duncan, R. A., Lim, J., Nelson, G. J., Drake, S. A., \& Kundu, M. R. 1994, ApJ, 429, 380.

White, S. M., Welch, W. J., Vogel, S. N., \& Lim, J. 1998, ApJ, in preparation.

Williams, P. M., van der Hucht, K. A., Pollock, A. M. T., Florkowski, D. R., van der Woerd, H., \& Wamsteker, W. M. 1990, MNRAS, 243, 662.

Wright, A. E., \& Barlow, M. J. 1975, MNRAS, 170, 41. 